Section: PHAGOCYTES

\title{
Derivation of functional mature neutrophils from human embryonic stem cells
}

Yasuhisa Yokoyama, ${ }^{1,2,6}$ Takahiro Suzuki, ${ }^{1,2,4,7}$ Mamiko Sakata-Yanagimoto, ${ }^{1,2,6}$ Keiki Kumano, ${ }^{1,2}$ Katsumi Higashi, ${ }^{3}$ Tsuyoshi Takato, ${ }^{4}$ Mineo Kurokawa, ${ }^{2}$ Seishi Ogawa, ${ }^{1,4,5}$ and Shigeru Chiba ${ }^{1,6}$

${ }^{1}$ Department of Cell Therapy and Transplantation Medicine, University of Tokyo Hospital, Tokyo, Japan; ${ }^{2}$ Department of Hematology and Oncology, Graduate School of Medicine, University of Tokyo, Tokyo, Japan; ${ }^{3}$ Department of Clinical Hematology, School of Health Sciences, Kyorin University, Tokyo, Japan; ${ }^{4}$ Divison of Tissue Engineering, University of Tokyo Hospital, Tokyo, Japan; ${ }^{5}$ The 21 st Century COE Program, Graduate School of Medicine, University of Tokyo, Tokyo, Japan; ${ }^{6}$ Department of Clinical and Experimental Hematology, University of Tsukuba, Ibaraki, Japan

Current affiliation: ${ }^{7}$ Department of Hematology, Jichi Medical University, Tochigi, Japan

Correspondence: Shigeru Chiba, Department of Clinical and Experimental Hematology, University of Tsukuba, 1-1-1 Tennodai, Tsukuba, Ibaraki, 305-8575, Japan; e-mail: schiba-tky@umin.net; phone: +81-29-853-3103; FAX: +81-29-853-3127

text: 4994 words; abstract: 199 words; 5 figures and 1 table; 40 references 


\begin{abstract}
Human embryonic stem cells (hESC) proliferate infinitely and are pluripotent. Only a few reports, however, describe specific and efficient methods to induce hESC to differentiate into mature blood cells. It is important to determine whether and how these cells, once generated, behave similarly with their counterparts produced in vivo. We developed a method to induce the differentiation of hESC into mature neutrophils. Embryoid bodies were formed with bone morphogenic protein-4, stem cell factor (SCF), Flt-3 ligand (FL), interleukin (IL) -6/IL-6 receptor fusion protein (FP6), and thrombopoietin (TPO). The cells derived from the embryoid bodies were cultured on a layer of irradiated OP9 cells with the combination of SCF, FL, FP6, IL-3, and TPO, which was later changed to granulocyte-colony stimulating factor. Morphologically mature neutrophils were obtained in approximately 2 weeks with a purity and efficiency sufficient for functional analyses. The population dominantly containing mature neutrophils (hESC-Neu) showed superoxide production, phagocytosis, bacterial killing, and chemotaxis, similar to peripheral blood neutrophils from normal subjects, although there were differences in the surface antigen expression patterns, such as lower CD16, and aberrant CD64 and CD14 expression in hESC-Neu. Thus, this is the first description of a detailed functional analysis of mature hESC-derived neutrophils.
\end{abstract}




\section{Introduction}

Embryonic stem (ES) cells can self-renew and differentiate into cells derived from all three germ layers, i.e., ectoderm, endoderm, and mesoderm. Both mouse and human ES cells give rise to mature blood cells of granulocyte/macrophage, erythroid, and megakaryoid lineages in vitro. For blood cell induction from ES cells, the majority of investigators use a co-culturing system with mouse stromal cells such as $\mathrm{S} 17^{1}$ or OP9. ${ }^{2,3}$ Embryoid body (EB) formation is also a commonly used method to obtain starting materials for further culture. ${ }^{4-6}$ Cell surface antigens, such as CD45 and CD34, and colony-forming ability are used as blood cell markers. The production of hemangioblasts, which have the capacity to differentiate into both endothelial and blood cells, has also been documented..$^{7-9}$ Only a few studies, however, have achieved specific and effective induction of mature blood cells from ES cells, particularly human ES cells (hESC). ${ }^{10}$

Human ESC-derived blood cells are potentially useful as a replacement for donation-based blood for transfusion in clinical settings, for drug discovery screening, and for monitoring drug efficacy and toxicity. The current blood donation system for transfusion is incapable of establishing granulocyte transfusion to patients with life-threatening neutropenia, although granulocyte transfusion could have a potentially significant benefit for a certain population of severely neutropenic patients. ${ }^{11,12}$ Given the large amount of neutrophils required for transfusion, ${ }^{13}$ hESC-derived neutrophils might be a unique solution for this treatment demand. Therefore, the development of an extremely effective method of neutrophil differentiation from hESC is an important step for both clinical application of hESC and granulocyte transfusion medicine.

The lack of an effective method for obtaining hESC-derived neutrophils with purity sufficient for functional analysis, however, has hampered progress in this field. Once neutrophils with a high purity are generated from hESC, it will be important to determine whether and how they behave similarly to and differently from neutrophils produced in vivo, particularly given the fact that hESC rarely give rise to hematopoietic stem cells in vitro, ${ }^{14}$ and thus, that hESC-derived neutrophils might not be a progeny of hematopoietic stem cells. Here, we developed an effective method of deriving mature neutrophils from hESC through EB formation and subsequent co-culture with OP9, and analyzed their morphologic and phenotypic characteristics. We then performed functional analysis of hESC-derived neutrophils in vitro, focusing on superoxide production, phagocytosis, bacterial killing, and chemotaxis, in comparison with peripheral blood neutrophils separated from normal subjects.

\section{Materials and Methods}

\section{Human ES cell culture and EB formation}

In all experiments using hESC, we used KhES-3 ${ }^{15}$ cells (a kind gift from Dr. Nakatsuji; Kyoto University, Kyoto, Japan), which were maintained as described previously. ${ }^{16}$ Briefly, KhES-3 colonies were cultured on irradiated mouse embryonic fibroblasts in Dulbecco's modified Eagle's medium/F12 
(Invitrogen, Carlsbad, CA) supplemented with 20\% KNOCKOUT serum replacer (Invitrogen) and 2.5 $\mathrm{ng} / \mathrm{mL}$ human basic fibroblast growth factor (Invitrogen). The culture medium was replaced daily with fresh medium. Colonies were passaged onto new mouse embryonic fibroblasts every 6 days. To induce the formation of EBs, KhES-3 colonies were picked up using collagenase, and cultured in suspension in non-serum stem cell medium that we previously used in a hematopoietic stem cell expansion protocol. ${ }^{17}$ After 24 hours, the colonies formed EBs, which were collected and cultured further for 17 days in Iscove's modified Dulbecco's medium (IMDM) (Invitrogen) containing 15\% fetal bovine serum (FBS), 1\% non-essential amino acid (Invitrogen), $2 \mathrm{mM} \mathrm{L-glutamine,} 100 \mathrm{U} / \mathrm{mL}$ penicillin, $100 \mu \mathrm{g} / \mathrm{mL}$ streptomycin, and $0.1 \mathrm{mM}$ 2-ME supplemented with cytokines $[25 \mathrm{ng} / \mathrm{mL}$ bone morphogenic protein-4 (R\&D Systems, Minneapolis, MN), $50 \mathrm{ng} / \mathrm{mL}$ stem cell factor (SCF; R\&D Systems), $50 \mathrm{ng} / \mathrm{mL}$ Flt-3 ligand (FL; R\&D Systems), $50 \mathrm{ng} / \mathrm{mL}$ interleukin (IL) -6/IL-6 receptor fusion protein (FP6; Kirin Pharma, Tokyo, Japan), and 20 ng/mL thrombopoietin (TPO; Kirin Pharma)].

\section{Expansion of hematopoietic progenitor cells and terminal differentiation into mature neutrophils on OP9 stromal cells}

OP9 cells (a kind gift from Dr. Nakano; Osaka University, Osaka, Japan) were irradiated with 20 Gy and plated onto a gelatin-coated 6-well tissue culture plate at a density of $1.5 \times 10^{5} /$ well. The next day, the EBs (incubated for 18 days from the initiation of suspension culture) were trypsinized and disrupted into single cells. Cells were suspended in the progenitor expansion medium [IMDM supplemented with 10\% FBS, 10\% horse serum (Stem Cell Technologies, Vancouver, BC, Canada), 5\% protein-free hybridoma medium (Invitrogen), $0.1 \mathrm{mM}$ 2-ME, $100 \mathrm{U} / \mathrm{mL}$ penicillin, $100 \mu \mathrm{g} / \mathrm{mL}$ streptomycin, $100 \mathrm{ng} / \mathrm{mL} \mathrm{SCF}$, FL, FP6, and $10 \mathrm{ng} / \mathrm{mL}$ TPO and IL-3 (R\&D Systems)] and plated onto the irradiated OP9 cells (day 0). Each well contained up to $5 \times 10^{5}$ EB-derived cells. The culture medium was replaced with fresh medium on day 4.

On day 7 of the progenitor expansion phase, floating cells were collected, suspended with terminal differentiation medium [IMDM supplemented with 10\% FBS, $0.1 \mathrm{mM} 2-\mathrm{ME}, 100 \mathrm{U} / \mathrm{mL}$ penicillin, $100 \mu \mathrm{g} / \mathrm{mL}$ streptomycin, and $50 \mathrm{ng} / \mathrm{mL}$ granulocyte colony-stimulating factor (G-CSF; Kirin Pharma)], and transferred onto the newly irradiated OP9 cells. The culture medium was replaced with fresh medium on day 10. This terminal differentiation phase culture was continued for 6 or 7 days.

\section{Preparation of normal peripheral blood neutrophils and bone marrow mononuclear cells}

Human peripheral blood and bone marrow cells were obtained from normal healthy adult donors after the informed consent was obtained. Peripheral blood neutrophils were prepared as previously described. ${ }^{18}$ The purity of the neutrophils was greater than $90 \%$, with the remaining cells mainly eosinophils. Neutrophils were suspended in Hank's balanced salt solution (HBSS; Invitrogen) containing $0.5 \%$ bovine serum albumin (BSA) and placed at $4{ }^{\circ} \mathrm{C}$. In some experiments, peripheral blood mononuclear 
cells (PB-MNC) were collected from the intermediate layer after centrifugation with Lymphoprep. Bone marrow cells were directly centrifuged with Lymphoprep, and only mononuclear cells were collected. Bone marrow-mononuclear cells (BM-MNC) were immediately used for RNA extraction.

\section{Wright-Giemsa, myeloperoxidase, and alkaline-phosphatase stainings}

The morphology of and granule characteristics in hESC-derived cells at the indicated days were assessed by Wright-Giemsa staining, comparing them with normal peripheral blood neutrophils (PB-Neu). Myeloperoxidase (MPO) and alkaline-phosphatase staining were performed using the New PO-K staining kit and alkaline phosphatase staining kit (MUTO PURE CHEMICALS, Tokyo, Japan). The prepared slides were inspected using an Olympus BX51 microscope equipped with a 100×/1.30 uPlan objective lens (Olympus, Tokyo, Japan). Images were acquired with an HC-2500 digital camera and Photograb-2500 software (Fujifilm, Tokyo, Japan).

\section{Electron microscopy}

The population after 13- or 14-day culture dominantly contained morphologically mature neutrophils, and was defined as hESC-Neu. The hESC-Neu and PB-Neu were fixed in $2 \%$ paraformaldehyde $/ 2.5 \%$ glutaraldehyde in $0.1 \mathrm{M}$ phosphate buffered saline (PBS; Invitrogen) for at least 12 hours, and then postfixed in $1 \%$ osmium tetroxide in $0.1 \mathrm{M}$ PBS for 2 hours. After fixation, samples were dehydrated in a graded ethanol series, cleared with propylene oxide, and embedded in Epon. Thin sections of cured samples were stained with uranyl acetate and Reynolds lead citrate. The sections were inspected using a transmission electron microscope, H7000 (Hitachi, Tokyo, Japan).

\section{Semiquantitative RT-PCR for lactoferrin}

Total RNA was obtained from hESC-derived cells of indicated culture days, PB-Neu, PB-MNC, and BM-MNC using RNeasy mini kit (QIAGEN, Hilden, Germany), and cDNA was synthesized from each RNA sample using random primer and SuperScript III (Invitrogen) following manufacturer's protocol. Semiquantitative PCR was performed as previously described. ${ }^{19}$ The sequence information of gene-specific primers used in RT-PCR and PCR conditions can be requested.

\section{Flow cytometric analysis}

Surface antigens of hESC-derived cells harvested on the indicated days were analyzed by flow cytometry using FACS Aria (Becton Dickinson Immunocytometry Systems, San Jose, CA). Fc receptors on the cells were blocked with PBS containing 2\% FBS and FcR Blocking Reagent (Myltenyi Biotec, Bergisch Gladbach, Germany). For staining of each antigen, fluorescein isothiocyanate-conjugated anti-human CD13, CD64, CD11b (Beckman Coulter, Fullerton, CA), and CD14 (BD Pharmingen, San Diego, CA) antibody, 
phycoerythrin-conjugated anti-human CD16, CD32, CD33 (Beckman Coulter), CD11b, and CD45 (BD Pharmingen) antibody, allophycocyanin-conjugated anti-human CD15, CD117 (BD Pharmingen), CD34, and CD133 (Myltenyi Biotec) were used. The negative range was determined by referencing the fluorescence of isotype controls. Dead cells were detected using 7-amino-actinomycin D (Via-Probe; BD Pharmingen).

\section{$\underline{\text { Apoptosis assay }}$}

Neutrophils (hESC-Neu and PB-Neu) were suspended in IMDM with $0.5 \%$ BSA and incubated in 6-well plates at $37^{\circ} \mathrm{C}$ with $5 \% \mathrm{CO}_{2}$, with or without $50 \mathrm{ng} / \mathrm{mL}$ of G-CSF. At the indicated time, neutrophils were harvested, stained with FITC-conjugated Annexin V and propidium iodide (PI) using Annexin V FITC Kit (Beckman Coulter), and analyzed by FACS Aria. Cells negative for both Annexin V and PI were judged as alive.

\section{G-CSF stimulation prior to assessing neutrophil function}

Because the functions of hESC-Neu are modified by G-CSF in the culture medium, we stimulated hESC-Neu and PB-Neu [PB-Neu(G+)] for 15 minutes at $37^{\circ} \mathrm{C}$ with $50 \mathrm{ng} / \mathrm{mL}$ of G-CSF in the reaction medium. As a control, PB-Neu without G-CSF stimulation [PB-Neu(G-)] was prepared. hESC-Neu, PB-Neu(G+), and PB-Neu(G-) were used for functional assays directly without changing the medium.

\section{Detection of reactive oxygen species produced by neutrophils}

Production of reactive oxygen species by neutrophils was detected by flow cytometry using dihydrorhodamine123 (DHR; Sigma-Aldrich, St Louis, MO) as described previously. ${ }^{20-22}$ Briefly, $1 \times 10^{5}$ hESC-Neu, PB-Neu(G+), or PB-Neu(G-) were suspended in $400 \mu \mathrm{L}$ of the reaction medium (HBSS containing $0.5 \%$ BSA) per tube, and 3 tubes were prepared of each sample. Catalase (Sigma-Aldrich) at a final concentration of $1000 \mathrm{U} / \mathrm{mL}, 1.8 \mu \mathrm{L} 29 \mathrm{mM} \mathrm{DHR}$, and $100 \mu \mathrm{L} 3.2 \mu \mathrm{M}$ phorbol myristate acetate (PMA; Sigma-Aldrich) were added to 1 of the 3 tubes; in the other 2 tubes, either no DHR or only DHR was added as controls. Reaction medium was added to bring the final volume up to $500 \mu \mathrm{L}$. After 15 minutes reaction at $37^{\circ} \mathrm{C}$, the samples were washed twice with ice-cold reaction medium, and suspended in $200 \mu \mathrm{L}$ reaction medium. Rhodamine fluorescence from the oxidized form of DHR was detected using the FACS Aria.

\section{Phagocytosis and NBT-reduction test using NBT-coated yeast cells}

Phagocytosis and NBT-reduction were visualized in a single set of experiments. Autoclaved Baker's yeast was suspended in $0.5 \%$ NBT solution [0.5\% NBT (Sigma-Aldrich) and 0.85\% sodium chloride in distilled water] at a density of $1 \times 10^{8} / \mathrm{mL}$. A $5-\mu 1$ aliquot of this yeast suspension was added to hESC-Neu, PB-Neu(G+), and PB-Neu(G-) at $2.5 \times 10^{5}$ in $50 \mu \mathrm{L}$ FBS. After 1-hour reaction at $37^{\circ} \mathrm{C}$, the 
samples were washed and stained with 1\% safranin-O (MUTO PURE CHEMCALS) for 5 minutes. The samples were then washed twice and suspended in $100 \mu \mathrm{L}$ PBS. A small aliquot of each sample was placed onto a glass slide and topped with a cover glass, and the number of ingested yeast cells and their change in color from brown to purple or black were examined using a microscope. Ingested yeast cells that changed color in the cells were counted as NBT-reaction positive, whereas those that were ingested but did not change color were counted as NBT-reaction negative. The phagocytosis rate was calculated as the percentage of neutrophils that contained one or more NBT-positive yeast cells. The phagocytosis score was calculated as the total number of positive yeast cells in 100 neutrophils. Only morphologically-determined neutrophils were scored, excluding contaminating cells such as macrophages, the percentage of which was less than $15 \%$ of the total cells.

\section{Bacterial killing assay}

Bacterial killing assay was performed using E. coli ATCC25922 as previously described ${ }^{23}$ with some modifications. Briefly, $1 \times 10^{8}$ colony-forming units (CFU) of exponentially growing bacteria were suspended in $1 \mathrm{~mL}$ of HEPES-buffered saline with $10 \%$ human AB serum (MP Biomedicals, Irvine, CA) and opsonized at $37^{\circ} \mathrm{C}$ for 30 minutes. Neutrophils [hESC-Neu, PB-Neu(G-) and (G+)] were suspended in HEPES-buffered saline with $40 \%$ human $\mathrm{AB}$ serum at a concentration of $5 \times 10^{6} / \mathrm{mL}$. The opsonized $E$. coli was added to the suspension of hESC-Neu and PB-Neu, at the neutrophil/bacteria ratio of $2: 1$, or control medium. After 1 hour incubation, $50 \mu \mathrm{L}$ of samples with and without neutrophils were diluted in 2.5 $\underline{\mathrm{mL}}$ alkalinized water $(\mathrm{pH} 11)$ for lysis of neutrophils. Samples were further diluted with PBS, and duplicates of aliquots were added to molten tryptic soy broth with $1.5 \%$ agar kept at $42^{\circ} \mathrm{C}$, rapidly mixed, and plated on dishes. The CFU were counted after overnight incubation.

\section{Chemotaxis assay}

Chemotactic ability was determined using a modified Boyden chamber method. ${ }^{24}$ Briefly, $700 \mu \mathrm{L}$ of the reaction medium (HBSS containing $5 \% \mathrm{BSA}$ ) with or without $10^{-7} \mathrm{M}$ formyl-Met-Leu-Phe (fMLP; Sigma-Aldrich) was placed into each well of a 24 -well plate, and the cell culture insert (3.0- $\mu \mathrm{m}$ pores; Falcon, Becton Dickinson, Franklin Lakes, NJ) was gently placed into each well to divide the well into upper and lower sections. Neutrophils were suspended in the reaction medium at $2.5 \times 10^{6} / \mathrm{mL}$ and $200 \mu \mathrm{L}$ cell suspension was added to the upper well, allowing the neutrophils to migrate from the upper to the lower side of the membrane for 90 minutes at $37^{\circ} \mathrm{C}$. After incubation, the membranes were washed, fixed with methanol, stained with Carrazi's hematoxylin (MUTO PURE CHEMICALS), and mounted on the slide glass. The number of neutrophils that migrated through the membrane from the upper to the lower side was counted using a microscope with a high-power lens $(\times 400)$ at three fields; two near the edge and one on the center. Only mature neutrophils were counted. 


\section{Statistical analyses}

Results are expressed as mean \pm SD. Statistical significance was determined using a two-tailed Student $t$ test. Results were considered significant when $P$ values were less than 0.05 .

Results

\section{Effective derivation of mature neutrophils from hESC with high purity}

After initiating the suspension culture of EB-derived cells, small clusters of round-shaped cells appeared around day 4 on the OP9 stromal layer. The morphology of the cells visualized with Wright-Giemsa staining of the day 7 population suggested that they were myeloblasts and promyelocytes. On days 9 and 11, myelocytes and metamyelocytes were dominant, and on day 13 or 14, $70 \%$ to $80 \%$ of the cells appeared to be stab and segmented neutrophils (Figure 1므), with about $90 \%$ of the granulocytes at the metamyelocyte stage or later (Table 1). This finding indicated that hESC-derived cells differentiated into mature neutrophils by a process similar to physiologic granulopoiesis. The remaining cells appeared to be macrophages or monocytes, and cells of other lineages, such as erythroid or lymphoid cells, did not appear at any time during the culture. The number of total cells peaked around days 9 to 11 , with an average 2.9-fold increase (range; 0.5-10.0-fold in 23 independent cultures) compared with the initial EB-derived cell number. The final yield of the cells on day 13 or 14 was 1.7 -fold (range; $0.1-8.8$ in 28 independent cultures). We attempted whether we could further purify hESC-derived mature neutrophils from the "hESC-Neu" population by density gradient methods. We, however, were unable to succeed in achieving higher purification without massively reducing cell yield. We used "hESC-Neu" in the subsequent experiments.

Most $(97.3 \pm 1.5 \%)$ of the Wright-Giemsa staining-proven hESC-derived mature neutrophils were positive for MPO staining, and alkaline-phosphatase score of hESC-Neu was $284 \pm 8.6$ (Figure 1B ). Under transmission electron microscopy, segmented nuclei and round cytoplasmic granules of hESC-Neu appeared very similar to those in PB-Neu (Figure 1C).

Some myeloid cell lines, such as HL-60, have abnormalities in biosynthesis of second granule proteins. ${ }^{25,26}$ Thus, it is important to examine biosynthesis of the second granule proteins in hESC-Neu. We examined lactoferrin mRNA in hESC-derived cells at different stages with PB-Neu and BM-MNC by semiquantitative RT-PCR (Figure 1D). Lactoferrin biosynthesis begins at myelocyte stage and terminates by

the beginning of the band stage. ${ }^{25,27}$ We found that lactoferrin mRNA was not detected in PB-Neu from some donors but was detected in PB-Neu from others. Human ESC-derived cells at various stages as well as BM-MNC expressed lactoferrin mRNA. The expression level of lactoferrin mRNA in the hESC-derived cells was the highest at day 10 of the induction culture and declined at days 13-14. These findings were compatible with the documented pattern of lactoferrin biosynthesis. 


\section{Surface antigen presentation in comparison to peripheral blood neutrophils}

Surface antigen expression at each level of differentiation of hESC-derived cells was analyzed by flow cytometry (Figure 2). From day 7 to day 13, the common blood cell antigen CD45 was expressed in almost all the cells. CD34, CD117, and CD133, cell surface markers on normal immature hematopoietic cells, were detected in a small population of the cells on day 7 , but disappeared by day 10 . Common myeloid antigens CD33 and CD15 were also highly expressed, while the CD11b expression level increased during the course of maturation. CD13 is also a common myeloid antigen, but its expression was observed in less than $20 \%$ of the cells on day 7 and did not increase afterward. CD16 [Fc $\gamma$ receptor (Fc $\gamma \mathrm{R}$ ) III], which is expressed in neutrophils as well as natural killer cells, macrophages, and a small subset of monocytes, ${ }^{28}$ was already expressed by day 7, and increased with maturation. This expression pattern of CD16 is consistent with that during normal neutrophil differentiation, although the proportion of CD16-positive cells was lower than that of mature neutrophils, as determined by morphology, on day 13 . The ratio of CD32 (Fc $\gamma$ RII)-positive cells increased as differentiation stage advanced, and eventually reached 90\%. CD64 (Fc $\gamma \mathrm{RI}$ ) expression was greater than $80 \%$, peaking on day 10 , and the high percentage was maintained through day 13 . CD14 was expressed in $20 \%$ to $25 \%$ of the cells on days 10 and 13 .

In normal peripheral blood, both neutrophils and monocytes express CD15 and CD11b. In addition, mature neutrophils express CD16, while monocytes express CD14. ${ }^{28,29}$ Detailed analysis on day 13 revealed that approximately $70 \%$ of CD15 and CD11b double-positive cells were CD16 positive, and almost all CD15 and CD16 double-positive cells expressed CD11b (Figure 2Bi, ii). Given that $70 \%$ to $80 \%$ of the cells on day 13 were morphologically mature neutrophils (Table 1), it is likely that the majority of hESC-Neu had CD15, CD11b, and CD16 expression patterns similar to PB-Neu, although some hESC-Neu did not express CD15 or CD16, particularly CD16.

CD32 is broadly expressed on myeloid cells, while CD64 is expressed only on monocytes but not on neutrophils in the peripheral blood. ${ }^{28}$ In the bone marrow, CD64 expression is observed in a small population of myeloblasts, peaks at the promyelocyte, myelocyte and metamyelocyte stages, and diminishes afterward, although a small part of the stab neutrophils still express CD64. ${ }^{30,31}$ We confirmed that virtually no PB-Neu expressed CD64 (data not shown). In contrast, almost all CD15 and CD16 double-positive hESC-Neu expressed CD64 on day 13, indicating that both stab and segmented hESC-Neu expressed CD64, because segmented neutrophils represented more than $50 \%$ of the cells on day 13 (Figure 2Biii and Table 1). Nearly 50\% of CD15 and CD16 double-positive cells were weakly positive for CD14, which is dissimilar to the negative expression of CD14 in steady-state peripheral blood neutrophils (Figure 2Biv). This aberrant expression of CD64 and CD14 in hESC-Neu is similar to their positive expression on some of the neutrophils harvested from healthy donors who received G-CSF administration, ${ }^{32,33}$ and the neutrophils derived from bone marrow CD34-positive cells in vitro by G-CSF stimulation. ${ }^{31}$

\section{$\underline{\text { Apoptosis pattern and prolongation of survival by G-CSF of hESC-Neu and PB-Neu }}$}


In the steady state, peripheral blood neutrophils have a short life span of approximately 24 hours, but this can be prolonged by G-CSF stimulation. ${ }^{34}$ We found that some hESC-Neu were already apoptotic at the time of the harvest, and thus, focused on the non-apoptotic fraction of hESC-Neu (Figure 3). In contrast to the PB-Neu that underwent apoptosis within 6 hours without G-CSF, which was compatible with the description in the literature, the proportion of apoptotic cells did not increase up to 6 hours after the start of the culture of hESC-Neu in the medium without G-CSF. In addition, there were no differences between the cultures with and without G-CSF up to 6 hours. After 6 hours, non-apoptotic cells in hESC-Neu without G-CSF decreased more rapidly than hESC-Neu with G-CSF, resulting in the lower number of viable cells than hESC-Neu with G-CSF at 24 hours, although the number of viable cells of hESC-Neu without G-CSF was still higher than that of PB-Neu without G-CSF.

\section{Oxidative burst phenotype was similar in hESC-Neu and PB-Neu.}

Oxidative burst is an essential function of neutrophils to kill microorganisms, whereas an inappropriate burst sometime causes injury to the host tissues. We assessed the ability to convert DHR to rhodamine in hESC-Neu and PB-Neu using flow cytometry. ${ }^{20}$ Because G-CSF, which could substantially affect the result, was used during the culture, we compared hESC-Neu with PB-Neu(G+) and PB-Neu(G-) as described in the "Materials and Methods." When DHR was added to the neutrophil suspensions, rhodamine-specific fluorescence was detected in hESC-Neu, PB-Neu(G-) and (G+) without PMA stimulation, indicating basal superoxide production without PMA stimulation in each neutrophil preparation (Figure 4). PMA stimulation increased rhodamine MFI in hESC-Neu though in less extent than in

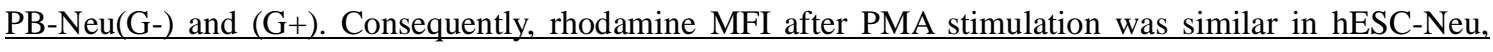
PB-Neu(G-) and (G+), suggesting that the maximum ability of the superoxide production in hESC-Neu and PB-Neu is comparable with each other.

\section{Phagocytosis and NBT reduction activities, and bactericidal ability were similar between hESC-Neu and PB-Neu.}

Neutrophils protect us from infectious microorganisms by phagocytosing and subsequently killing them. These functions in hESC-Neu and PB-Neu were evaluated in an experimental system using NBT-coated yeast. Under the microscope, mature neutrophils could be easily distinguished from contaminating macrophages by their unique shape of nuclei after the $1 \%$ safranin-O staining (Figure $\underline{5 \mathrm{~A}}$ ). NBT-coated yeast that had not been ingested were colored red-brown; they began to change their color to purple or black, beginning at the periphery, and eventually became completely black, because the NBT coating on the yeast was reduced by neutrophils after phagocytosis. This enabled us to identify neutrophils that had phagocytosis and NBT-reducing ability. hESC-Neu had a slightly lower phagocytosis rate than PB-Neu(G-) and (G+) (Figure 5B). The phagocytosis score, however, was not significantly different between hESC-Neu and PB-Neu(G-) and (G+) (Figure $\underline{5 \mathrm{C}})$. The cells on day 8 of the culture, most of which 
were morphologically myeloblasts and promyelocytes, were rarely observed to phagocytose the yeast or reduce NBT if they had ingested the yeast, indicating that we observed phagocytosis and NBT reduction that was specific to mature neutrophils.

Since hESC-Neu have sufficient ability of phagocytosis and superoxide production, we next investigated whether hESC-Neu can actually kill bacteria. Bactericidal activity of hESC-Neu comparing with PB-Neu was evaluated using E. coli. When incubated with hESC-Neu and PB-Neu(G-) and (G+), the numbers of CFU were similarly reduced to approximately $40 \%$ of the control, indicating that the killing ability against E.coli is comparable between hESC-Neu and PB-Neu (Figure 5D).

\section{Chemotaxis was similar between hESC-Neu and PB-Neu.}

We compared chemotaxis of hESC-Neu and PB-Neu using a modified Boyden chamber method. After incubation with or without fMLP in the lower well, neutrophils had migrated from the upper side to the lower side of the membrane. Neutrophil migration without fMLP in the lower well was considered random migration. The number of neutrophils that migrated randomly was not significantly different between hESC-Neu and PB-Neu(G-), but PB-Neu(G+) showed significantly more random migration than the others (Figure $\underline{5 \mathrm{E}}$ ). The number of migrated cells increased in all hESC-Neu, PB-Neu(G-), and PB-Neu(G+) when fMLP was added in the lower well. The increase of the migrated cells by chemotaxis to fMLP was calculated by subtracting the number of randomly migrated cells without fMLP from that of migrated cells with fMLP. There were no significant differences between hESC-Neu and PB-Neu(G-) or $(\mathrm{G}+)$ in the net fMLP-induced chemotaxis.

\section{Discussion}

We developed a method of specific derivation of mature neutrophils from hESC, and this effective method made it possible to analyze hESC-derived neutrophils in detail. hESC-derived neutrophils had characteristics similar to steady-state peripheral blood mature neutrophils in morphology and essential functions, although there were some differences in surface antigen expression.

We intended further purification of hESC-derived mature neutrophils from the "hESC-Neu" population by density gradient methods, but this lead to massive reduction of the cell yield. When analyzed using flow cytometer, the mean intensity of hESC-Neu in forward scatter was higher than that of PB-Neu (data not shown), indicating that the size of morphologically mature neutrophils, which represent $70-80 \%$ of the hESC-Neu population, was larger than PB-Neu. This fact further indicates that the density of morphologically mature neutrophils in the hESC-Neu population is lower than that of PB-Neu, which makes separation of hESC-Neu from contaminating other cells difficult.

In this culture, we observed morphologically defined myeloblasts, promyelocytes, myelocytes, metamyelocytes, and, eventually, mature stab and segmented neutrophils in this order during the 13-day culture, which is similar to the granulocyte maturation process in the bone marrow. The surface antigen 
expression pattern during differentiation was similar to that during normal granulopoiesis, with the expression of CD34 and CD117 on immature cells, and an increase in CD16 as differentiation advanced. Most hESC-Neu expressed CD16, CD15, CD11b, CD33, and CD45. This pattern is consistent with normal peripheral blood neutrophils, but the percentage of CD16-expressing cells was lower than that of mature neutrophils determined by morphology. The lower CD16 expression level is documented in neutrophils derived in vitro from bone marrow CD34-positive cells by stimulation with G-CSF, and is considered to be the effect of G-CSF on myeloid progenitors. ${ }^{31}$ G-CSF also induces CD64 and CD14 on mature neutrophils, ${ }^{31,35}$ and these effects are also observed in vivo when G-CSF is administered to healthy volunteers. ${ }^{32,33}$ Therefore, it is possible that the G-CSF present in the culture from day 7 affected the progenitors and led to the relatively low expression of CD16 on hESC-Neu and aberrant expression of CD64 and CD14 on CD15 and CD16 double-positive hESC-Neu.

In the apoptosis assay, some hESC-Neu were already apoptotic at the time of the harvest from the induction culture, but the proportion of apoptotic cells did not increase up to 6 hours after the start of the culture of hESC-Neu in the medium without G-CSF. We raised two reasons that might explain the difference in the manner of apoptosis. First, hESC-Neu are more heterogeneous than PB-Neu, as containing cells at different stages, from incompletely differentiated cells such as metamyelocytes to maturation-completed and aged neutrophils. Relatively immature cells or unaged mature neutrophils in the hESC-Neu population might have longer lifetime than PB-Neu. Second, the effect of G-CSF used in the induction culture might remain even after the washout.

In the chemotaxis assay, the random migration of hESC-Neu was almost the same as that of PB-Neu(G-), but lower than that of PB-Neu(G+), despite the fact that hESC-Neu were stimulated by G-CSF before the assay. The effect of G-CSF on the random migration of neutrophils is controversial; random migration increases in vitro when neutrophils are stimulated by G-CSF, ${ }^{36}$ whereas neutrophils obtained from G-CSF-treated patients with non-myeloid malignancies show decreased random migration and chemotaxis. ${ }^{37,38}$ Our in vitro experiment with $\mathrm{PB}-\mathrm{Neu}(\mathrm{G}+)$ and $\mathrm{PB}-\mathrm{Neu}(\mathrm{G}-)$ reproduced the former result. Nevertheless, hESC-Neu showed relatively low random migration despite stimulation with G-CSF, while maintaining almost normal fMLP-induced chemotaxis. One possible reason for these differences might be the continuous stimulation by G-CSF; hESC-Neu were stimulated from the myeloblast stage, and thus, it was expected that the characteristics of the hESC-Neu were more similar to those of neutrophils from G-CSF-stimulated donors rather than to normal mature neutrophils.

The low yield of hESC-Neu is a major obstacle to their functional analysis in animals, and further, for their potential use in drug screening and clinical applications. The number of hESC-Neu produced was less than twice that of input EB-derived cells. Recently, erythroid progenitor cell lines that could differentiate both in vitro and in vivo into functional mature red blood cells were established from mouse ESC. ${ }^{39}$ In that report, the starting number of ESC required to establish one progenitor line was $5 \times 10^{5}$, and transplantation of $2 \times 10^{7}$ cells of the progenitor line could ameliorate anemia in a mouse by increasing the red blood cell 
count. Similar methods could be considered in the granulopoiesis from human ESC. Another potential method is to use more immature or proliferation-competent cells than the cells we initiated the induction culture. One of the candidates is hematopoietic progenitors that emerge in sac-like structures derived from ESC. In a report using cynomolgus monkey ESC, ${ }^{40} \mathrm{~EB}$ were created and subsequently subjected to adherent culture on gelatin-coated dish. After two weeks, the sac-like structures emerged, with hematopoietic precursors inside. The cells inside the sac consisted of various stages of myeloid lineage cells. The authors also mention the possibility of existence of hemangioblasts, because endothelial cells could be produced from those precursors under a different condition. Others have also reported the similar sac-like structures containing hematopoietic precursors created from hESC. ${ }^{10}$ In this paper, megakaryocytes were created from the inner cells, which were positive for hematoendothelial markers, such as CD34, CD31, VEGF-receptor 2 , and VE-cadherin. These similar findings indicate that the cells in the sac-like structures might contain more immature cells than our EB-derived cells, and that the precursors inside the sac-like structures may have greater proliferation potency than our EB-derived cells. Because either paper did not directly demonstrate the efficiency of mature blood cell production from monkey or human ES cells, however, it is of worth to compare the efficiency of producing neutrophils from our EB-derived cells with that from the sac-like structure-derived cells.

\section{Acknowledgments}

We thank Dr. Nakatsuji for providing the KhES-3, and Dr. Nakano for providing the OP9 cells. We are grateful to Kirin Pharma for providing TPO, FP6, and G-CSF, and Kyokuto Pharmaceutical Industrial for the non-serum medium used in the EB formation. We also thank S. Ichimura for hESC culture. This work was supported in part by a Grant-in-aid from the Japan Society of Promotion of Sciences (KAKENHI \# 17390274) and a Grant-in-aid from the Ministry of Health, Welfare, and Labor of Japan.

\section{Authorship}

Contribution: Y.Y. and T.S. performed the experiments; K.H. designed the NBT-coated yeast cell-phagocytosis test; M.S.-Y, and K.K. assisted with interpretation of experiments and provided insightful comments; Y.Y. interpreted the data, made the figures, and wrote the manuscript; T.T., M.K., and S.O. advised on experimental design; S.C. provided critical reading of the manuscript; T.S. and S.C. designed the research.

Conflict-of-interest disclosure: The authors declare no competing financial interests.

Correspondence: Shigeru Chiba, Department of Clinical and Experimental Hematology, University of Tsukuba, 1-1-1 Tennodai, Tsukuba, Ibaraki, 305-8575, Japan; e-mail: schiba-tky@umin.net.

\section{References}

1. Kaufman DS, Hanson ET, Lewis RL, Auerbach R, Thomson JA. Hematopoietic colony-forming 
cells derived from human embryonic stem cells. Proc Natl Acad Sci U S A. 2001;98:10716-10721.

2. Nakano T, Kodama H, Honjo T. Generation of lymphohematopoietic cells from embryonic stem cells in culture. Science. 1994;265:1098-1101.

3. Vodyanik MA, Bork JA, Thomson JA, Slukvin, II. Human embryonic stem cell-derived CD34+ cells: efficient production in the coculture with OP9 stromal cells and analysis of lymphohematopoietic potential. Blood. 2005;105:617-626.

4. Chadwick K, Wang L, Li L, et al. Cytokines and BMP-4 promote hematopoietic differentiation of human embryonic stem cells. Blood. 2003;102:906-915.

5. Cerdan C, Rouleau A, Bhatia M. VEGF-A165 augments erythropoietic development from human embryonic stem cells. Blood. 2004;103:2504-2512.

6. Wang L, Menendez P, Shojaei F, et al. Generation of hematopoietic repopulating cells from human embryonic stem cells independent of ectopic HOXB4 expression. J Exp Med. 2005;201:1603-1614.

7. Keller G, Kennedy M, Papayannopoulou T, Wiles MV. Hematopoietic commitment during embryonic stem cell differentiation in culture. Mol Cell Biol. 1993;13:473-486.

8. Wang L, Li L, Shojaei F, et al. Endothelial and hematopoietic cell fate of human embryonic stem cells originates from primitive endothelium with hemangioblastic properties. Immunity. 2004;21:31-41.

9. Lu SJ, Feng Q, Caballero S, et al. Generation of functional hemangioblasts from human embryonic stem cells. Nat Methods. 2007;4:501-509.

10. Takayama N, Nishikii H, Usui J, et al. Generation of functional platelets from human embryonic stem cells in vitro via ES-sacs, VEGF-promoted structures that concentrate hematopoietic progenitors. Blood. 2008;111:5298-5306.

11. Hubel K, Carter RA, Liles WC, et al. Granulocyte transfusion therapy for infections in candidates and recipients of HPC transplantation: a comparative analysis of feasibility and outcome for community donors versus related donors. Transfusion. 2002;42:1414-1421.

12. Mousset S, Hermann S, Klein SA, et al. Prophylactic and interventional granulocyte transfusions in patients with haematological malignancies and life-threatening infections during neutropenia. Ann Hematol. 2005;84:734-741.

13. Price TH. Granulocyte transfusion: current status. Semin Hematol. 2007;44:15-23.

14. Bhatia M. Hematopoietic development from human embryonic stem cells. Hematology Am Soc Hematol Educ Program. 2007;2007:11-16.

15. Suemori H, Yasuchika K, Hasegawa K, Fujioka T, Tsuneyoshi N, Nakatsuji N. Efficient establishment of human embryonic stem cell lines and long-term maintenance with stable karyotype by enzymatic bulk passage. Biochem Biophys Res Commun. 2006;345:926-932.

16. Thomson JA, Itskovitz-Eldor J, Shapiro SS, et al. Embryonic stem cell lines derived from human blastocysts. Science. 1998;282:1145-1147.

17. Suzuki T, Yokoyama Y, Kumano K, et al. Highly efficient ex vivo expansion of human 
hematopoietic stem cells using Delta1-Fc chimeric protein. Stem Cells. 2006;24:2456-2465.

18. Yuo A, Kitagawa S, Okabe T, et al. Recombinant human granulocyte colony-stimulating factor repairs the abnormalities of neutrophils in patients with myelodysplastic syndromes and chronic myelogenous leukemia. Blood. 1987;70:404-411.

19. Kumano K, Chiba S, Shimizu K, et al. Notch1 inhibits differentiation of hematopoietic cells by sustaining GATA-2 expression. Blood. 2001;98:3283-3289.

20. Vowells SJ, Sekhsaria S, Malech HL, Shalit M, Fleisher TA. Flow cytometric analysis of the granulocyte respiratory burst: a comparison study of fluorescent probes. J Immunol Methods. 1995;178:89-97.

21. Richardson MP, Ayliffe MJ, Helbert M, Davies EG. A simple flow cytometry assay using dihydrorhodamine for the measurement of the neutrophil respiratory burst in whole blood: comparison with the quantitative nitrobluetetrazolium test. J Immunol Methods. 1998;219:187-193.

22. Emmendorffer A, Hecht M, Lohmann-Matthes ML, Roesler J. A fast and easy method to determine the production of reactive oxygen intermediates by human and murine phagocytes using dihydrorhodamine 123. J Immunol Methods. 1990;131:269-275.

23. Decleva E, Menegazzi R, Busetto S, Patriarca P, Dri P. Common methodology is inadequate for studies on the microbicidal activity of neutrophils. J Leukoc Biol. 2006;79:87-94.

24. Harvath L, Falk W, Leonard EJ. Rapid quantitation of neutrophil chemotaxis: use of a polyvinylpyrrolidone-free polycarbonate membrane in a multiwell assembly. J Immunol Methods. 1980;37:39-45.

25. Rado TA, Bollekens J, St Laurent G, Parker L, Benz EJ, Jr. Lactoferrin biosynthesis during granulocytopoiesis. Blood. 1984;64:1103-1109.

26. Rado TA, Wei XP, Benz EJ, Jr. Isolation of lactoferrin cDNA from a human myeloid library and expression of mRNA during normal and leukemic myelopoiesis. Blood. 1987;70:989-993.

27. Cowland JB, Borregaard N. The individual regulation of granule protein mRNA levels during neutrophil maturation explains the heterogeneity of neutrophil granules. J Leukoc Biol. 1999;66:989-995. 28. van de Winkel JG, Anderson CL. Biology of human immunoglobulin G Fc receptors. J Leukoc Biol. 1991;49:511-524.

29. van Lochem EG, van der Velden VH, Wind HK, te Marvelde JG, Westerdaal NA, van Dongen JJ. Immunophenotypic differentiation patterns of normal hematopoiesis in human bone marrow: reference patterns for age-related changes and disease-induced shifts. Cytometry B Clin Cytom. 2004;60:1-13.

30. Ball ED, McDermott J, Griffin JD, Davey FR, Davis R, Bloomfield CD. Expression of the three myeloid cell-associated immunoglobulin G Fc receptors defined by murine monoclonal antibodies on normal bone marrow and acute leukemia cells. Blood. 1989;73:1951-1956.

31. Kerst JM, van de Winkel JG, Evans AH, et al. Granulocyte colony-stimulating factor induces $\mathrm{hFc}$ gamma RI (CD64 antigen)-positive neutrophils via an effect on myeloid precursor cells. Blood. 1993;81:1457-1464.

32. Kerst JM, de Haas M, van der Schoot CE, et al. Recombinant granulocyte colony-stimulating factor administration to healthy volunteers: induction of immunophenotypically and functionally altered neutrophils 
via an effect on myeloid progenitor cells. Blood. 1993;82:3265-3272.

33. Carulli G. Effects of recombinant human granulocyte colony-stimulating factor administration on neutrophil phenotype and functions. Haematologica. 1997;82:606-616.

34. van Raam BJ, Drewniak A, Groenewold V, van den Berg TK, Kuijpers TW. Granulocyte colony-stimulating factor delays neutrophil apoptosis by inhibition of calpains upstream of caspase-3.

Blood. 2008;112:2046-2054.

35. Kerst JM, Slaper-Cortenbach IC, von dem Borne AE, van der Schoot CE, van Oers RH. Combined measurement of growth and differentiation in suspension cultures of purified human CD34-positive cells enables a detailed analysis of myelopoiesis. Exp Hematol. 1992;20:1188-1193.

36. Nakamae-Akahori M, Kato T, Masuda S, et al. Enhanced neutrophil motility by granulocyte colony-stimulating factor: the role of extracellular signal-regulated kinase and phosphatidylinositol 3-kinase. Immunology. 2006;119:393-403.

37. Azzara A, Carulli G, Rizzuti-Gullaci A, Minnucci S, Capochiani E, Ambrogi F. Motility of rhG-CSF-induced neutrophils in patients undergoing chemotherapy: evidence for inhibition detected by image analysis. Br J Haematol. 1996;92:161-168.

38. Ribeiro D, Veldwijk MR, Benner A, et al. Differences in functional activity and antigen expression of granulocytes primed in vivo with filgrastim, lenograstim, or pegfilgrastim. Transfusion. 2007;47:969-980. 39. Hiroyama T, Miharada K, Sudo K, Danjo I, Aoki N, Nakamura Y. Establishment of mouse embryonic stem cell-derived erythroid progenitor cell lines able to produce functional red blood cells. PLoS ONE. 2008;3:e1544.

40. Nakahara M, Matsuyama S, Saeki K, et al. A feeder-free hematopoietic differentiation system with generation of functional neutrophils from feeder- and cytokine-free primate embryonic stem cells. Cloning Stem Cells. 2008;10:341-354. 
Table

Table 1. Differentiation pattern of hESC-derived cells.

\begin{tabular}{lccc}
\hline \multirow{2}{*}{\multicolumn{1}{c}{ Cell type }} & \multicolumn{3}{c}{ \% of total cells } \\
\cline { 2 - 4 } & Day 7 & Day 10 & Day 13 \\
\hline Myeloblast & $61.0 \pm 9.1$ & $2.3 \pm 1.2$ & ND \\
Promyelocyte & $16.8 \pm 6.3$ & $8.5 \pm 0.9$ & $0.7 \pm 0.8$ \\
Myelocyte & $12.3 \pm 4.8$ & $34.0 \pm 6.8$ & $6.4 \pm 3.4$ \\
Metamyelocyte & $3.0 \pm 1.0$ & $19.0 \pm 1.3$ & $10.2 \pm 4.3$ \\
Stab neutrophil & $0.8 \pm 0.3$ & $16.2 \pm 3.0$ & $18.3 \pm 2.6$ \\
Segmented neutrophil & $0.3 \pm 0.6$ & $14.7 \pm 6.0$ & $53.1 \pm 9.6$ \\
Macrophage/monocyte & $5.7 \pm 0.6$ & $5.3 \pm 1.3$ & $11.2 \pm 1.4$ \\
Mature neutrophil & $1.2 \pm 0.8$ & $30.8 \pm 4.6$ & $71.4 \pm 7.4$ \\
\hline
\end{tabular}

Mature neutrophil is the total of the stab and segmented neutrophils. Data are shown as mean \pm SD $(n=3$ independent experiments). ND indicates not detectable.

\section{Figure Legends}

Figure 1. Morphology of hESC-derived cells and expression of lactoferrin mRNA.

(A) Wright-Giemsa staining of the day 7 population (i) revealed that they were morphologically myeloblasts and promyelocytes. On days 9 (ii) and 11 (iii), myelocytes and metamyelocytes were dominant, and on day 13 (hESC-Neu) (iv), 70-80\% of the cells appeared to be stab and segmented neutrophils. Original magnification, $\times 1000$. (B) (i) MPO staining revealed that $97.3 \pm 1.5 \%$ of hESC-Neu were MPO-positive. (ii) The neutrophil alkaline-phosphatase score in hESC-Neu was $284 \pm 8.6$. Values represent mean \pm SD. Original magnification, $\times 1000$. (C) Microstructure of hESC-Neu. Similar to steady-state neutrophils separated from peripheral blood (i), segmented nucleus and cytoplasmic granules were observed in hESC-Neu (ii). Original magnification, $\times 8000$. (D) Lactoferrin (LTF) mRNA was already expressed in

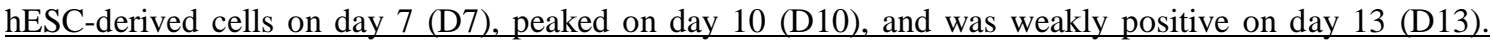
Bone marrow mononuclear cells (BM) were strongly positive for LTF mRNA, but peripheral blood neutrophils (PB1 and 2) were negative, although faint bands were detected in peripheral blood neutrophils prepared from some donors (data not shown). As a negative control, peripheral blood mononuclear cells (MNC) were used.

Figure 2. Surface antigens of hESC-derived cells. 
(A) Surface antigen expression at each level of differentiation of hESC-derived cells was analyzed by flow cytometry. CD45 was expressed in almost all the cells. CD34, CD117, and CD133, immature markers of hematopoiesis, were detected in a small population of the cells on day 7, and had almost disappeared by day 10. Common myeloid antigens CD33 and CD15 were highly expressed, and the expression of CD11b increased during maturation. CD13 was expressed in less than $20 \%$ of the cells throughout the culture period. The expression of CD16, a mature neutrophil marker, increased following maturation, but was observed in only approximately $45 \%$ of the cells, even on day 13. CD14 and CD64 expression was aberrantly observed in some cells. Bars represent SDs $(n=3)$. (B) In the steady state, mature neutrophils in peripheral blood are CD15, CD11b, and CD16 positive. (i) In hESC-derived cells on day $13,63.3 \pm 2.6 \%$ of the CD15- and CD11b-positive cells were CD16 positive, and (ii) almost all of the CD15 and CD16-positive cells were CD11b-positive. (iii-iv) On the other hand, CD64 and CD14 were rarely expressed on mature neutrophils in the peripheral blood. CD15 and CD16-positive cells from hESC, consistent with the phenotype of mature neutrophils, showed aberrant expression of CD64 (iii) and CD14 (iv), in $94.1 \pm 3.8 \%$ and $45.1 \pm 9.6 \%$ of the cells, respectively. Data are presented as mean $\pm \mathrm{SD}(\mathrm{n}=3)$.

\section{Figure 3. Apoptosis pattern and G-CSF effect on survival of hESC-Neu}

(A) Flowcytometric analysis. In the steady state, peripheral blood neutrophils have a short life span of approximately 24 hours, but this can be prolonged by G-CSF stimulation (a-e). Some hESC-Neu were already apoptotic at the time of the harvest from the induction culture (f). In contrast to the PB-Neu that underwent apoptosis within 6 hours without G-CSF (b), the proportion of apoptotic cells did not increase up to 6 hours after the start of the culture of hESC-Neu in the medium without G-CSF (f, g). In addition, there were no differences between the cultures of hESC-Neu with and without G-CSF up to 6 hours (g). After 6 hours, non-apoptotic cells in hESC-Neu without G-CSF decreased more rapidly than hESC-Neu with G-CSF (h-j), resulting in the lower number of viable cells than hESC-Neu with G-CSF at 24 hours (j). Figures are representative of 3 independent experiments. Data are presented as mean $\pm S D(n=3)$. (B) The $\underline{\text { time course of decrease in viable cells. Bars represent SDs }(n=3)}$.

\section{Figure 4. Superoxide production of hESC-Neu assessed by dihydrorhodamine123 oxidation.}

(A) Dihydrorhodamine123 (DHR) was reacted to neutrophils with or without phorbol myristate acetate (PMA), and the resultant rhodamine fluorescence was detected by flow cytometry. When DHR was added to the reaction medium without PMA (line), the fluorescence levels were slightly elevated in hESC-Neu (i), PB-Neu(G-) (ii), and PB-Neu(G+) (iii). The addition of PMA dramatically increased the levels of fluorescence in all three neutrophil preparations (bold line). The figures are representative of 3 independent experiments. (B) Comparison of superoxide production between hESC-Neu and PB-Neu using mean fluorescence intensity (MFI) of rhodamine. When DHR was added without PMA stimulation, rhodamine-specific fluorescence was detected in hESC-Neu, PB-Neu(G-) and (G+). PMA stimulation 
increased rhodamine MFI in hESC-Neu though in less extent than in PB-Neu(G-) and $(\mathrm{G}+)$. Consequently, rhodamine MFI after PMA stimulation was similar in hESC-Neu, PB-Neu(G-) and (G+), suggesting that the maximum ability of the superoxide production in hESC-Neu and PB-Neu is comparable with each other

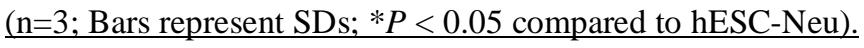

\section{Figure 5. NBT-coated yeast cell-phagocytosis test, bactericidal killing, and chemotaxis assay.}

(A) NBT-coated yeast cells were added to a neutrophil suspension, and incubated at $37^{\circ} \mathrm{C}$. After 1 hour, the cells were stained with $1 \%$ safranin-O, and observed using a microscope. Mature neutrophils (black arrow) could be easily distinguished from contaminating macrophages (white arrow; only the nucleus is observed in the figure) by the unique shape of their nuclei. Yeast cells were colored red-brown before being ingested (white arrow head); the color began to change to purple or black beginning at the periphery of the yeast cell, and eventually became completely black (black arrow head) because of reduced NBT after ingestion. Yeast cells that changed color in the cells were counted as NBT-reduction positive. Original magnification, $\times 400$. (B) The phagocytosis rate was calculated as a percentage of the neutrophils that contained one or more yeast cells. hESC-Neu had a slightly lower phagocytosis rate than that of PB-Neu(G-) and (G+). (C) The phagocytosis score was calculated as the total number of positive yeast cells in 100 neutrophils. There were no significant differences in the phagocytosis score between hESC-Neu and PB-Neu(G-) or (G+). The cells on day 8 of the culture (Day-8 cells) were rarely observed to phagocytose the yeast cells or reduce NBT [In panels $\mathrm{B}$ and $\mathrm{C}, \mathrm{n}=3$; bars indicate SDs; $* P<0.05$ compared to PB-Neu(G-) and $(\mathrm{G}+) ; * * P<0.05$ compared to hESC-Neu, PB-Neu(G-), and PB-Neu(G+)]. (D) Bactericidal assay. E. coli was opsonized with human $\mathrm{AB}$ serum, and incubated with hESC-Neu, PB-Neu(G-), PB-Neu(G+), or control medium. After 1 hour incubation with hESC-Neu, PB-Neu(G-), and PB-Neu(G+), the numbers of CFU were significantly reduced to approximately $40 \%$ of the control. There were no significant differences between hESC-Neu, PB-Neu(G-), and PB-Neu(G+) in bactericidal ability. The CFU of control are presented as $100 \%$ ( $\mathrm{n}=3$; Bars indicate SDs; $* P<0.05$ compared to control). (E) Chemotaxis assay by modified Boyden chamber method. The number of neutrophils that migrated randomly [fMLP(-)] was not different between hESC-Neu and PB-Neu(G-), but PB-Neu(G+) showed significantly greater random migration than hESC-Neu and PB-Neu(G-). The number of migrated cells increased in all hESC-Neu, PB-Neu(G-), and PB-Neu(G+) when fMLP was added to the lower well [fMLP(+)]. The increase in the number of migrated cells by chemotaxis to fMLP [fMLP(+)-fMLP(-)] was not significantly different between hESC-Neu and PB-Neu(G-) or $(\mathrm{G}+)(\mathrm{n}=3$; bars indicate SDs; $* P<0.05)$. 
Figures

Figure 1.

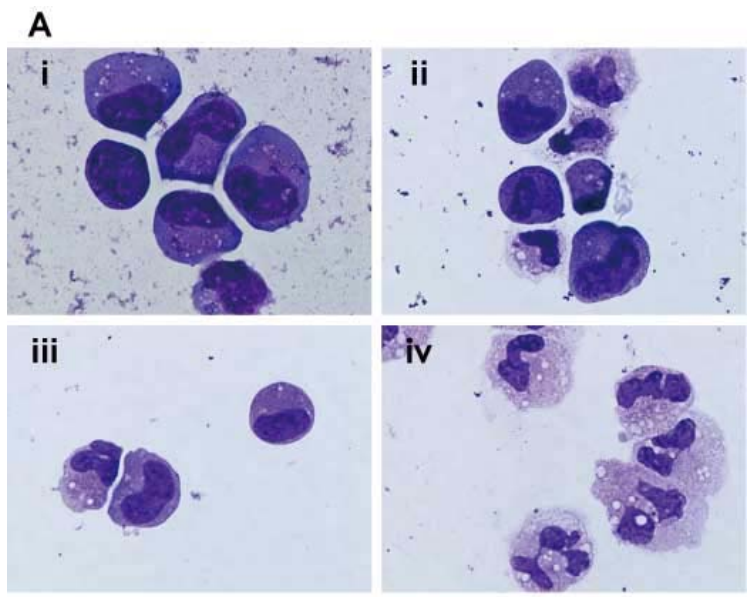

B
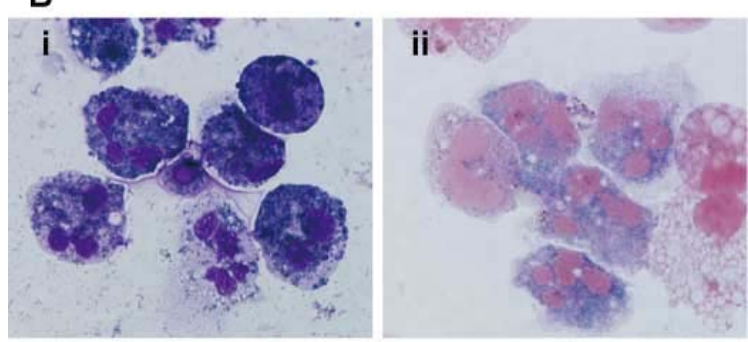

C

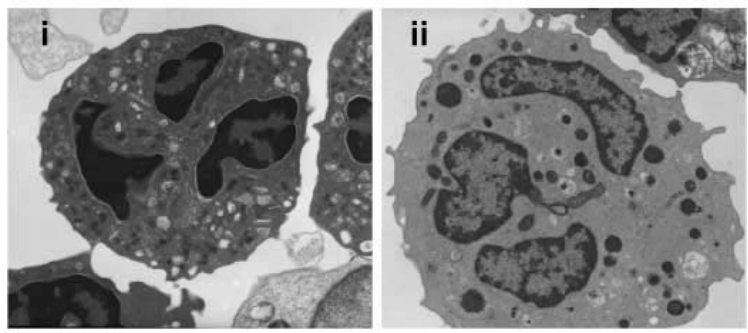

D

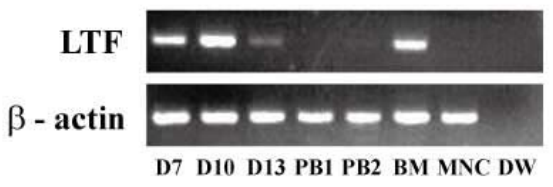


Figure 2.
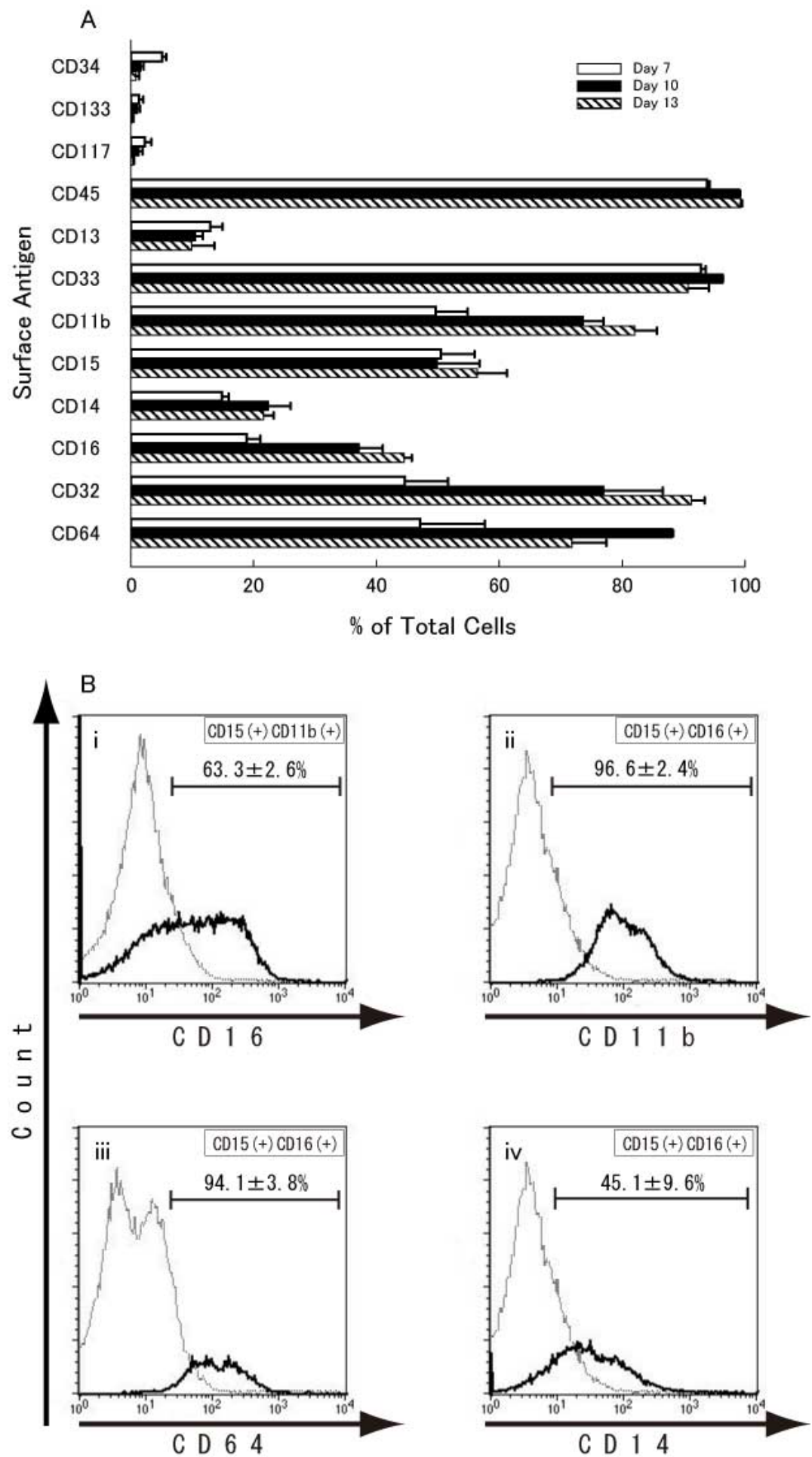
Figure 3.

\section{A}
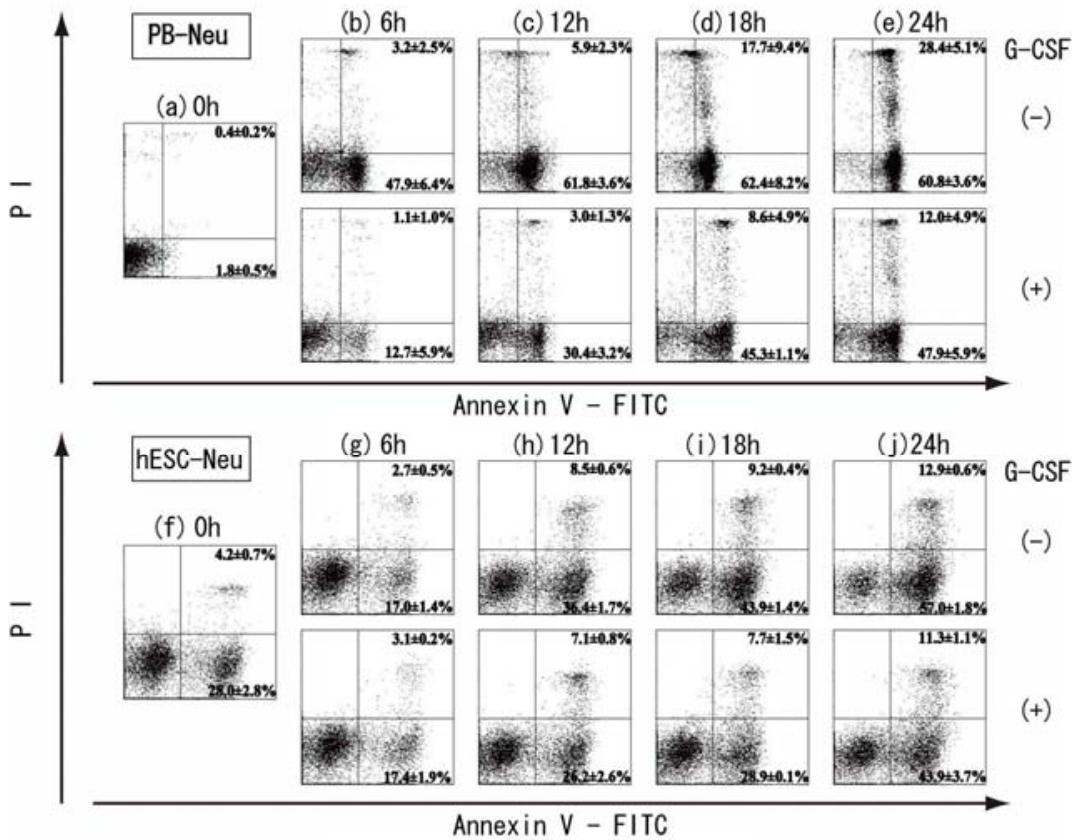

B

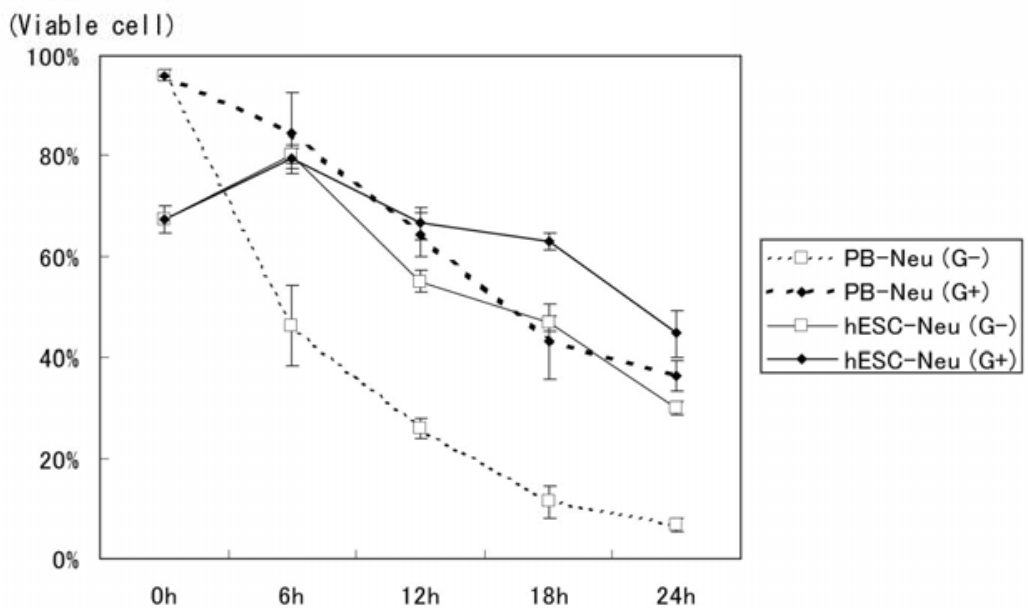


Figure 4.

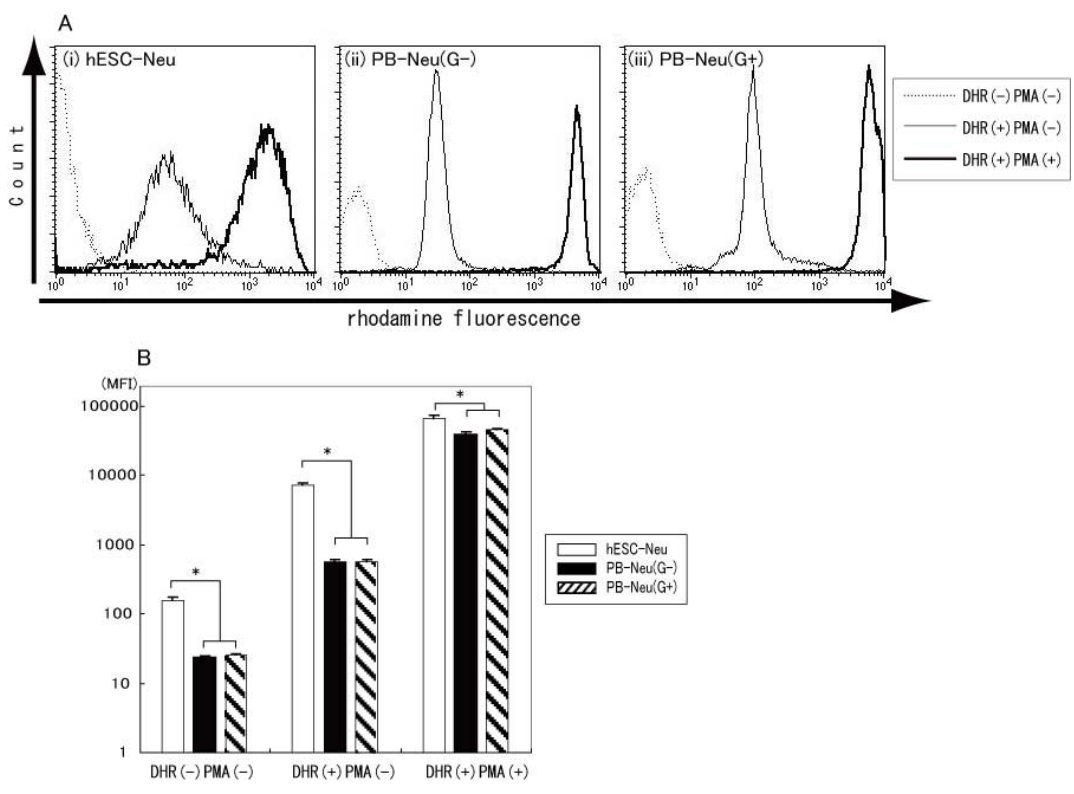


Figure 5.

A
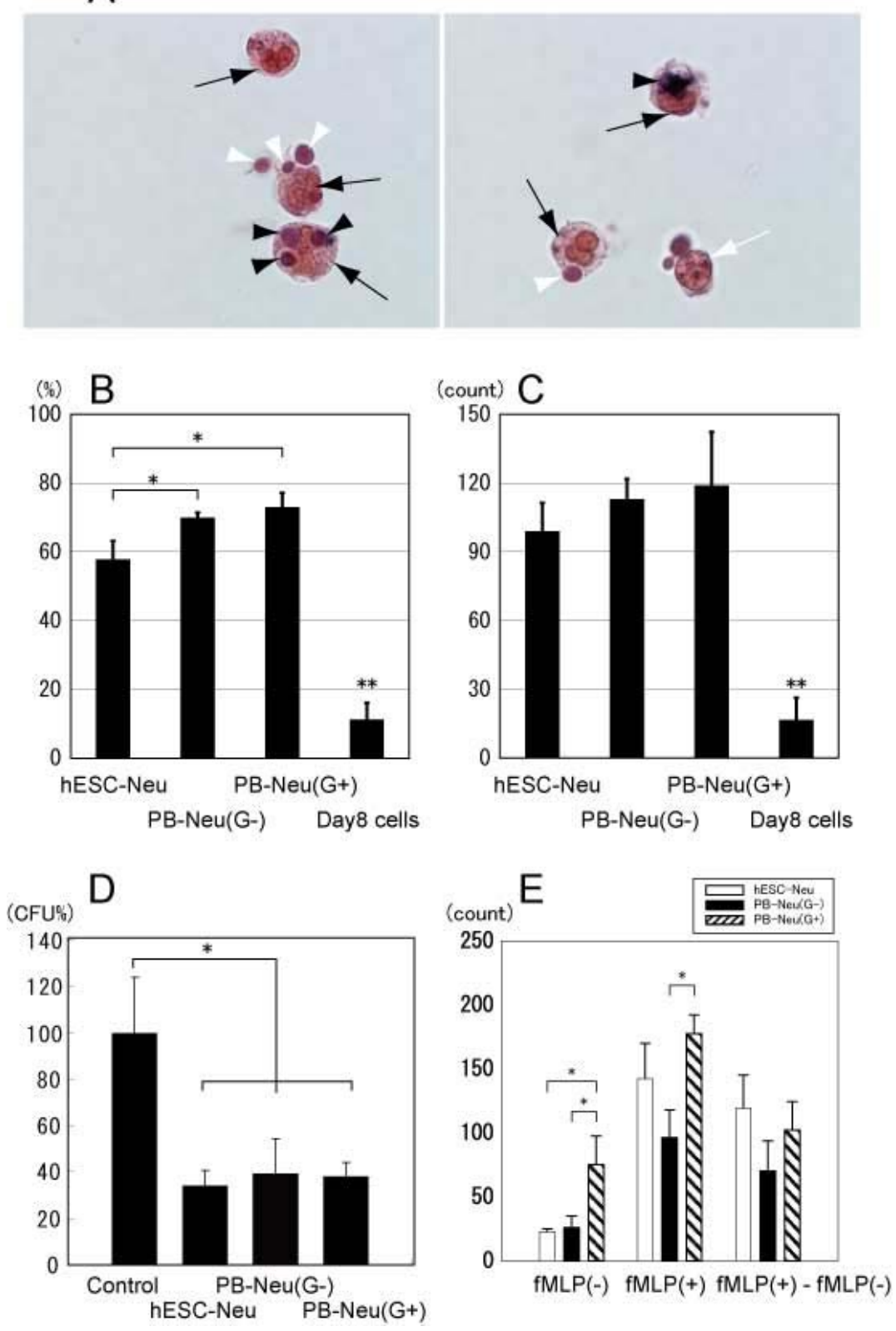